\title{
Social stress impact on developmental stability of laboratory rat Rattus norvegicus
}

\author{
Alexey V. VALETSKY, Irina L. DMITRIEVA, Natalia L. KRUSHINSKAYA \\ and Vladimir M. ZAKHAROV
}

\begin{abstract}
Valetsky A. V., Dmitrieva I. L., Krushinskaya N. L. and Zakharov V. M. 1997. Social stress impact on developmental stability of laboratory rat Rattus norvegicus. [In: Developmental homeostasis in natural populations of mammals: phenetic approach. V. M. Zakharov and A. V. Yablokov, eds]. Acta Theriologica, Suppl. 4: 27-32.

Developmental stability of the progeny of pregnant female rats Rattus norvegicus Berkenhout, 1769 kept under different conditions was studied. In two experimental groups, females were exposed to social stress by keeping them in crowded cages at different stages of pregnancy, from the 1st to the 15th and from the 16 th to the 21th days, respectively. In the control group, pregnant females were kept under normal conditions. Fluctuating asymmetry of 20 skull characters (number of foramina for small blood vessels and nerves) was used as a measure of developmental stability. Variance of the difference in foramen number between the left and right sides of the skull (l-r) as well as mean number of asymmetrical traits per individual revealed an increase of fluctuating asymmetry in both experimental groups compared with the control group. These results imply the deterioration of developmental stability under impact of social stress.
\end{abstract}

N. K. Koltzov Institute of Developmental Biology, Russian Academy of Sciences, Vavilov Str. 26, Moscow 117808, Russia

Key words: Rattus norvegicus, non-metric traits, fluctuating asymmetry, developmental stability, social stress

\section{Introduction}

Developmental homeostasis has been suggested as a sensitive estimate of an organism's condition. From the morphogenetic view-point, homeostasis is characterized by developmental stability measured by developmental noise or chance variation that could not be interpreted as a result of genotype or environmental dissimilarity. Intraindividual variation, as small non-directional differences between homologous structures that should be equal, provides us with an opportunity to distinguish the developmental noise. As has been shown, fluctuating asymmetry of various characters, even those uncorrelated with each other, could increase under the impact of stress. This indicates an opportunity to reveal changes in developmental stability through the study of the limited set of morphological characters (Mather 1953, Zakharov 1987, 1989). 
In this study, we tried to find out if social stress affects developmental stability. Social stress has already been suggested as a possible cause of disturbance of developmental stability revealed at the peak year in a cyclic population of small mammals (Zakharov et al. 1991). To get a more direct answer to the question we have modelled such stress in a laboratory strain of rats. To establish the deterioration of developmental stability under the impact of social stress seems to be important for two reasons. In a wide sense, it would be important evidence characterizing the disturbance of developmental stability as a non-specific reaction of an organism to any stress. In previous studies deterioration of developmental stability was registered under the stress impact of various chemical and physical factors that could be interpreted as a toxic effect (Siegel and Smookler 1973, Siegel and Doyle 1975, Doyle et al. 1977, Pankakoski et al. 1992). The social stress would be essential to establish the same reaction of an organism. In a narrow sense, it would be an experimental justification to interpret a decrease in developmental stability at the peak year in a cyclic population as a result of social stress. Negative correlation of developmental stability with density has already been established for a cyclic shrew population in central Siberia (Zakharov et al. 1991).

\section{Material and methods}

An experiment was conducted on laboratory rats Rattus norvegicus Berkenhout, 1769 of the strain "Tryon maze bright" (Wahlsten 1972). Social stress was modeled by keeping 2 pregnant females with 8 males in one cage $(32 \times 57 \times 20 \mathrm{~cm}$ ). In the first experiment (group 1) females were exposed to the stress during the first 15 days of pregnancy, and then each was kept in a separate cage. In the second experiment (group 2), each female was kept in a separate cage from the first day of pregnancy, but then they were exposed to the stress during the last 6 days of pregnancy (from the 16 th to the 21st days of pregnancy). Each female in the control group was kept in a separate cage the entire time of the pregnancy. Offspring in all groups were kept with the mother in a separate cage for the first 14 days and then were kept separately, not more than 6 individuals of one sex in each cage, for 4 months.

Developmental stability was studied in three samples of the offspring. Experimental material consists of two samples: 14 individuals (progeny of 3 females) in the first experiment, and 17 individuals (progeny of 4 females) in the second experiment. A control sample was represented by 40 individuals (progeny of 8 females).

Twenty bilateral skull foramina (apertures through the bone for nerves and small blood vessels) were used for morphological analysis (Fig. 1). No directional asymmetry, antisymmetry, or sexual dimorphism was found for these characters. None of the correlation was revealed among the characters studied per se, $(1+r)$ values, as well as between their asymmetries, $(l-r)$ values. It has been established through the calculation of the coefficient of correlation ( $r$ ) for all possible paired comparisons between the characters and their asymmetries.

Fluctuating asymmetry as difference in the number of foramina on the left and right sides of the skull (l-r) was used to assess developmental stability. Variance of (l-r) was calculated to estimate fluctuating asymmetry for each character. The mean number of asymmetrical characters per individual was used as an integrated measure of developmental stability.

The non-parametric sign-test was used for the intersample comparison of variances for the whole set of characters. An $F$-test was used for pairwise comparisons of the variances and $t$-test for the intergroup comparison of the mean number of asymmetrical characters per individual (Sokal and Rohlf 1981). 


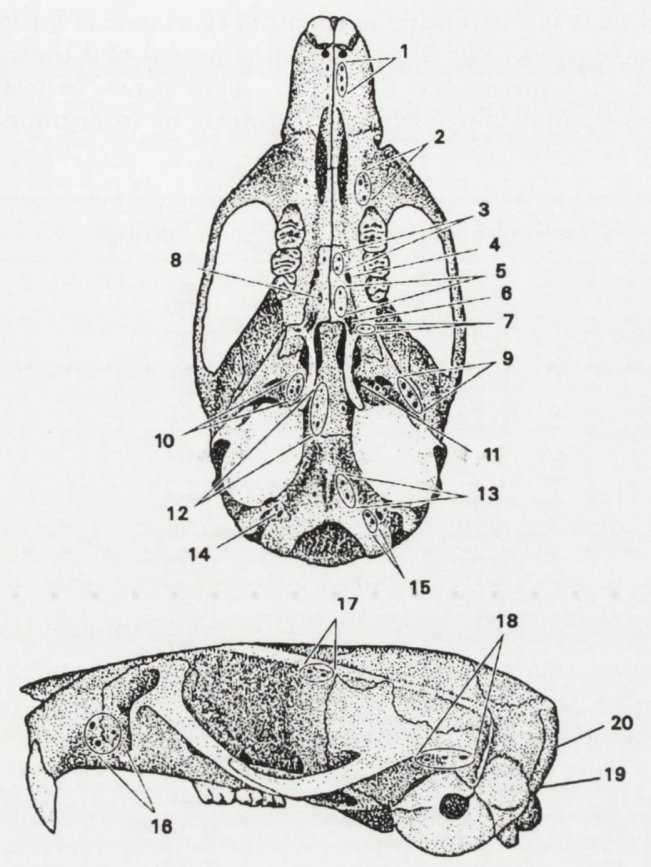

Fig. 1. Cranial characters (number of foramina) analyzed in the skull of the Norway rat Rattus norvegicus. 1 -foramen praemaxillaris ventralis, 2 -foramen diastemae, 3 -foramen palatinae minor anterior, 4 - foramen palatinae, 5 - foramen palatinae posterior minor I, 6 - foramen palatinae posterior minor II, 7 - foramen palatinae posterior minor III, 8 - foramen palatinum major, 9 - foramen pterigoideum, 10 - canalis transversalis accessorius, 11 -canalis transversalis, 12 - foramina basisphenoidalis lateralis, 13 - foramen basioccipitalis, 14 - foramen hypoglossum accessorius externus, 15 - foramen hypoglossum accessorius internus, 16 - foramen praemaxillaris lateralis, 17 foramen supraorbitalis, 18 - foramen postglenoidale, 19 - foramen occipitalis lateralis, 20 - foramen occipitalis medialis.

\section{Results}

Because of the limited material available in this study, we used the general intersample comparison. We have revealed neither the significant proportion of interlitter differences $(0.16 \pm 0.21)$ nor the significant difference between the higher intralitter variance (2.60) and smaller interlitter variance (2.17) by ANOVA $(F=1.20, \mathrm{df}=25,6, p>0.05)$ for the total experimental material (31 individuals of 7 litters).

Comparison of two experimental groups exposed to stress at different stages of pregnancy did not reveal significant differences between them in fluctuating asymmetry. Mean number of asymmetrical characters per individual (NA $\pm \mathrm{SE}$ ) proved to be similar in both experimental groups: $14.28 \pm 0.40$ in the first experimental group stressed during the first 15 days of pregnancy and $13.41 \pm 0.39$ 
Table 1 . The values of fluctuating asymmetry (variance of l-r for 20 characters of skull morphology, see Fig. 1) in the experimental and control groups of the Norway rat Rattus norvegicus. Statistical significance ( $F$-test) of intergroup difference is presented as $p$. Higher variance in intergroup comparison is underlined.

\begin{tabular}{rrrl}
\hline Character & Experimental group & Control group & \multicolumn{1}{c}{$p$} \\
\hline 1 & 1.00 & $\underline{1.60}$ & $\mathrm{~ns}$ \\
2 & $\underline{0.97}$ & 0.32 & $<0.01$ \\
3 & $\underline{0.57}$ & 0.02 & $<0.001$ \\
4 & $\underline{0.73}$ & 0.69 & $\mathrm{~ns}$ \\
5 & $\underline{1.18}$ & 0.02 & $<0.001$ \\
6 & $\underline{1.51}$ & 0.53 & $<0.01$ \\
7 & $\underline{0.70}$ & 0.36 & $<0.05$ \\
8 & $\underline{0.71}$ & 0.22 & $<0.001$ \\
9 & 1.76 & 0.81 & $<0.05$ \\
10 & $\underline{3.06}$ & 0.87 & $<0.001$ \\
11 & 1.46 & $\underline{1.52}$ & $\mathrm{~ns}$ \\
12 & 2.05 & 0.87 & $<0.001$ \\
13 & $\underline{2.77}$ & 1.99 & $\mathrm{~ns}$ \\
14 & 1.23 & 0.40 & $<0.01$ \\
15 & $\underline{1.09}$ & 0.26 & $<0.001$ \\
16 & 1.25 & 1.34 & $\mathrm{~ns}$ \\
17 & $\underline{1.18}$ & 0.22 & $\mathrm{n}$ \\
18 & 0.47 & $\underline{0.63}$ & $\mathrm{~ns}$ \\
19 & 2.53 & 2.19 & $\mathrm{~ns}$ \\
20 & $\underline{1.41}$ & 0.90 & $\mathrm{~ns}$ \\
\hline
\end{tabular}

in the second group stressed during the last 6 days of pregnancy ( $p>0.05, t$-test). Both groups had significantly higher mean number of asymmetric characters ( $p$ $<0.001)$ than in the control group $(9.07 \pm 0.34)$. This similarity allowed us to pool the two experimental groups into one sample to compare with the control group.

Variance of (l-r) was higher for the pooled experimental sample than for the control group in 16 of 20 characters under study ( $p<0.05$, sign-test). When treating the characters separately, significant differences $(p<0.05)$ were revealed for 12 characters (Table 1). Mean number of asymmetrical characters was significantly higher $(p<0.001)$ in the pooled experimental sample $(13.80 \pm 0.29)$ than in the control sample $(9.07 \pm 0.34)$.

\section{Discussion}

The general intersample comparison revealed neither the significant proportion of interlitter differences nor the significant difference between the higher intralitter variance and smaller interlitter variance for all experimental rat pups. These 
data corresponds to the conclusion established in many studies that heritability $\left(\mathrm{h}^{2}\right)$ of fluctuating asymmetry for various characters in different species, from insects to humans, does not differ from 0 (eg Reeve 1960, Bailit et al. 1970, Leary et al. 1985).

Stress impact of overpopulation was demonstrated in both natural populations and laboratory experiments (Christian and Davis 1964, Shilov 1977, Moshkin et al. 1990). Some biochemical, morphological and behavioral changes were observed for the progeny of females stressed during pregnancy (Thompson 1957, Naumenko et al. 1990, Pratt and Lisk 1990).

Our data on laboratory rats revealed an obvious developmental stability decrease in the progeny of females exposed to social stress at different stages of their pregnancy, comparing with a control group. The data indicate an adverse effect of stress on the pregnant female, and hence on the condition of her progeny. Previously, it was shown that prenatal and early postnatal stress from various physical factors could lead to decrease of developmental stability in mice and rats (Siegel and Smookler 1973, Siegel and Doyle 1975). In this study we demonstrate that social stress at different stages of prenatal development leads to the same adverse changes in an organism's condition that are revealed by a developmental stability decrease.

Thus, the study on the impact of social stress completes the previously obtained data on the impact of chemical and physical factors. Developmental stability disturbance seems to be a non-specific response of an organism to any stress impact. This study provides an experimental background to assess potential stress impact of overpopulation in population cycles of mammals. The results also support the assumption that developmental stability decrease during the peak year could be caused by the stressful impact of high density (Zakharov et al. 1991).

Acknowledgements: We are very grateful to Dr J. H. Graham and two anonymous referees provided very helpful comments and corrections of English.

\section{References}

Bailit H. L., Workman P. L., Niswander J. D. and Maclean C. J. 1970. Dental asymmetry as an indicator of genetic and environmental conditions in human populations. Human Biology 42: $626-638$.

Christian J. J. and Davis D. E. 1964. Endocrine, behavior, and population. Science 146: 1550-1560.

Doyle W. J., Kelley C. and Siegel M. I. 1977. The effects of audiogenic stress on the growth of long bones in the laboratory rat Rattus norvegicus. Growth 41: 183-189.

Leary R. F., Allendorf F. W. and Knudsen K. L. 1985. Inheritance of meristic variation and evolution of developmental stability in rainbow trout. Evolution 39: 308-314.

Mather K. 1953. Genetical control of stability in development. Heredity 7: 297-336.

Moshkin M. P., Gerlinskaya L. A. and Evsikov V. I. 1990. [Stress reactiveness and its adaptive role in population cycles of mammals.] [In: Ontogenetic and evolution-genetic aspects of neuroendocrinal regulation of stress. E. V. Naumenko, ed]. Nauka, Novosibirsk: 171-180. [In Russian]

Naumenko E. V., Dygalo N. N. and Maslova L. N. 1990. [Permanent modification of stress reactiveness caused by stressors in prenatal ontogeny.] [In: Ontogenetic and evolution-genetic 
aspects of neuroendocrinal regulation of stress. E. V. Naumenko, ed]. Nauka, Novosibirsk: 40-54. [In Russian]

Pankakoski E., Koivisto I. and Hyvärinen H. 1992. Reduced developmental stability as an indicator of heavy metal pollution in the common shrew Sorex araneus. Acta Zoologica Fennica 191: 137-144.

Pratt N. C. and Lisk R. D. 1990. Dexamethasone can prevent stress-related litter deficits in the golden hamster. Behavioral and Neural Biology 54: 1-12.

Reeve E. C. R. 1960. Some genetic tests on asymmetry of stenopleural chaeta number in Drosophila. Genetic Researches 1 (N1): 151-172.

Shilov I. A. 1977. [Ecophisiological base of population interaction of animals.] Moscow University Press, Moskva: 3-261. [In Russian]

Siegel M. I. and Doyle W. J. 1975. Stress and fluctuating limb asymmetry in various species of rodents. Growth 39: 363-369.

Siegel M. I. and Smookler H. H. 1973. Fluctuating dental asymmetry and audiogenic stress. Growth 37: $35-39$.

Sokal R. R. and Rohlf F. J. 1981. Biometry. Freeman, San-Francisco: 1-859.

Thompson W. R. 1957. Influence of prenatal maternal anxiety on emotionality in young rats. Science 125: 698-699.

Wahlsten D. 1972. Genetic experiments with animal learning: a critical review. Behavioral biology 7: 143-183.

Zakharov V. M. 1987. [Animal asymmetry: population-phenogenetic approach]. Nauka, Moskva: 1-216. [In Russian]

Zakharov V. M. 1989. Future prospects for population phenogenetics. Soviet Science Reviews, Section F 4 (3): 1-79.

Zakharov V. M., Pankakoski E., Sheftel B. I., Peltonen A. and Hanski I. 1991. Developmental stability and population dynamics in the common shrew, Sorex araneus. American Naturalist 138: 797-809.

Received 11 October 1995, revised 28 May 1997, accepted 1 July 1997. 